\title{
Erratum to: Forgotten Lives
}

\section{Katy Turton}

An incorrect content was temporarily assigned to this book. Since it has been corrected.

The correct content for this book is "[Forgotten Lives]" ([10.1057/9780230592209]).

The previously miss assigned contents belonged to [Oswald Mosley and the New Party] and its correct content is available under [http://link.springer.com/book/10.1057/9780230276529]

We apologize for this error. 